\title{
尖晶石型 $\mathrm{AFe}_{2} \mathrm{O}_{4}$ 催化剂的制备及其催化燃烧甲苯的研究
}

\author{
王永强 ${ }^{1,2}$, 薛 锐 ${ }^{1}$, 藏 萌 $^{1}$, 刘敏敏 ${ }^{1}$, 陈 䀬 ${ }^{1}$, 赵朝成 ${ }^{1,2}$
}

(中国石油大学(华东)1. 化学工程学院; 2. 石油石化污染物控制与处理国家重点实验室, 青岛 266580)

摘 要: 通过溶胶-凝胶一自蔓延燃烧法制备尖晶石型 $\mathrm{AFe}_{2} \mathrm{O}_{4}(\mathrm{~A}=\mathrm{Cu}, \mathrm{Co}, \mathrm{Ni}, \mathrm{Mg}, \mathrm{Zn})$ 催化剂, 以甲苯为 $\mathrm{VOCs}$ 模拟气, 考察 $\mathrm{AFe}_{2} \mathrm{O}_{4}$ 催化剂对 VOCs 的催化燃烧活性, 并采用 XRD、 $\mathrm{N}_{2}$ 吸附-脱附、SEM、TEM、 $\mathrm{H}_{2}-\mathrm{TPR} 、 \mathrm{XPS}$ 对催化 剂进行表征分析。结果表明: $\mathrm{AFe}_{2} \mathrm{O}_{4}$ 表现出较好的催化燃烧活性, 其中 $\mathrm{CuFe}_{2} \mathrm{O}_{4}$ 的催化燃烧活性最佳, 起燃温度 (T50)和完全燃烧温度 $(\mathrm{T} 90)$ 分别为 $188^{\circ} \mathrm{C} 、 239^{\circ} \mathrm{C} 。 \mathrm{AFe}_{2} \mathrm{O}_{4}$ 具有明显的片状尖晶石晶体, 并形成以介孔为主的多级 孔结构, 该特点为催化剂提供了大量表面活性位。 $\mathrm{A}$ 位元素种类对其催化燃烧活性影响较大, 当 $\mathrm{A}$ 位元素为 $\mathrm{Cu}$ 时, $\mathrm{Cu}$ 的 $\mathrm{H}_{2}$ 还原峰面积远大于其他元素, $\mathrm{H}_{2}$ 还原温度仅为 $289^{\circ} \mathrm{C}$, 表面亲电子氧和氧空位浓度占氧元素总量 $\left(\mathrm{O}_{\mathrm{el}} / \mathrm{O}_{1 \mathrm{~S}}\right)$ 的 $36 \%$ 。 $\mathrm{CuFe}_{2} \mathrm{O}_{4}$ 为片状反尖晶石晶型, 晶格体积仅为 $0.294 \mathrm{~nm}^{3}$, 并伴有 $\mathrm{CuO}$ 和 $\alpha-\mathrm{Fe}_{2} \mathrm{O}_{3}$ 物种。以介孔为主的多级 孔结构、特有的片状反尖晶石晶型以及该晶型与 $\mathrm{CuO}$ 和 $\alpha-\mathrm{Fe}_{2} \mathrm{O}_{3}$ 的协同作用是 $\mathrm{CuFe}_{2} \mathrm{O}_{4}$ 催化燃烧活性提高的主要 原因。

关 键 词: 尖晶石; VOCs; 催化燃烧; 铁酸盐

中图分类号: $\mathrm{O} 643$ 文献标识码: A

\section{Spinel $\mathrm{AFe}_{2} \mathrm{O}_{4}$ Catalysts: Preparation and Catalytic Combustion of Toluene}

\author{
WANG Yong-Qiang ${ }^{1,2}$, XUE Rui ${ }^{1}$, ZANG Meng $^{1}$, LIU Min-Min $^{1}$, CHEN Xi $^{1}$, ZHAO Chao-Cheng $^{1,2}$ \\ (1. College of Chemical Engineering, China University of Petroleum-Huadong, Qingdao 266580, China; 2. State Key Labora- \\ tory of Petroleum Pollution Control, China University of Petroleum-Huadong, Qingdao 266580, China)
}

\begin{abstract}
Nanocrystalline $\mathrm{AFe}_{2} \mathrm{O}_{4}(\mathrm{~A}=\mathrm{Cu}, \mathrm{Co}, \mathrm{Ni}, \mathrm{Mg}, \mathrm{Zn})$ ferrite spinel catalysts were prepared by Sol-Gel auto-combustion method. In order to investigate the influence of $\mathrm{A}$ sites in $\mathrm{AFe}_{2} \mathrm{O}_{4}$ spinel catalysts, the catalytic combustion of toluene was investigated, and the catalysts were characterized by XRD, $\mathrm{N}_{2}$ adsorption-desorption, SEM, TEM, $\mathrm{H}_{2}$-TPR, and XPS. It was found that $\mathrm{AFe}_{2} \mathrm{O}_{4}$ catalysts have excellent catalytic activity. Among the catalysts, the $\mathrm{CuFe}_{2} \mathrm{O}_{4}$ catalyst obtains the highest catalytic activity, and its light-off temperature $\left(T_{50}\right)$ and the complete combustion temperature $\left(T_{90}\right)$ of toluene were $188^{\circ} \mathrm{C}$ and $239^{\circ} \mathrm{C}$, respectively. And $\mathrm{AFe}_{2} \mathrm{O}_{4}$ catalysts have obviously lamellar spinel crystal structure and the multilevel pore structures with prevalence to mesoporous, which will improve the catalytic activity of the catalysts. It is concluded that the elements of A site on $\mathrm{AFe}_{2} \mathrm{O}_{4}$ have great influence on catalytic combustion activity. The $\mathrm{H}_{2}$ reduction temperature is only $289^{\circ} \mathrm{C}$, when A site was occupied by $\mathrm{Cu}$. $\mathrm{CuFe}_{2} \mathrm{O}_{4}$ has crystal lattice volume of $0.294 \mathrm{~nm}^{3}$ with $\mathrm{CuO}$ and $\alpha-\mathrm{Fe}_{2} \mathrm{O}_{3}$ formed in the crystal. The concentration of electrophilic oxygen and oxygen vacancy in all oxygens $\left(\mathrm{O}_{\text {ele }} / \mathrm{O}_{1 \mathrm{~s}}\right)$ is $36 \%$. Lamellar trans spinel crystal lattice, the synergies of trans spinel $\mathrm{CuFe}_{2} \mathrm{O}_{4}$ with $\mathrm{CuO}$ and $\alpha-\mathrm{Fe}_{2} \mathrm{O}_{3}$, and the multilevel pore structures with prevalence to mesoporous are the main reasons for the catalytic activity improvement of $\mathrm{CuFe}_{2} \mathrm{O}_{4}$.
\end{abstract}

收稿日期: 2016-12-29; 收到修改稿日期：2017-04-15

基金项目: 国家自然科学基金(51202294); 山东省自然科学基金(ZR2014EEM011)

National Natural Science Foundation of China (51202294); National Natural Science Foundation of Shandong Province (ZR2014EEM011)

作者简介: 王永强, 副教授. E-mail: wyqupc@163.com 
Key words: spinel; VOCs; catalytic combustion; ferrite

挥发性有机污染物(Volatile organic compounds, $\mathrm{VOCs}$ ) 是一类重要的大气污染物, 其种类多、来源 广、毒性强, 并且危害大。其中, 苯、甲苯、乙苯以 及临、间、对二甲苯等苯系物的危害更严重, 其 “三 致效应” 备受人类关注 ${ }^{[1]}$ 。VOCs 的治理方法主要有 吸附技术、微生物技术、光催化技术、低温等离子 体技术 ${ }^{[2]}$ 、催化燃烧技术等。其中, 催化燃烧技术以 其能耗低、处理效率高、无二次污染、处理彻底等 优点，被广泛应用于生产中 ${ }^{[3]}$ 。

催化燃烧催化剂种类繁多, 其中尖晶石催化剂 $\mathrm{AB}_{2} \mathrm{O}_{4}$ 具有特定结构, 正四面体和正八面体及其空 间群变化可以为催化剂提供大量氧空穴, 从而提高 其催化活性。其中铁酸盐催化剂 $\mathrm{AFe}_{2} \mathrm{O}_{4}$ 处于亚稳定 状态, 具有较高的化学势能, $\mathrm{Fe}$ 离子具有 +2 和 +3 两 种价态, 可以在正四面体和正八面体中转换, 晶格 畸变率高, 还原活性高 ${ }^{[4]}$ 。不同 $\mathrm{A}$ 位元素对 $\mathrm{AFe}_{2} \mathrm{O}_{4}$ 催化剂的催化活性影响较大, 常见的 $\mathrm{A}$ 位元素有 $\mathrm{Cu} 、 \mathrm{Co} 、 \mathrm{Ni} 、 \mathrm{Zn} 、 \mathrm{Mn}$ 等过渡金属元素，由于此类 元素具有可变价态，在掺杂过程中可以形成更多的 晶格缺陷, 从而表现出较好的催化活性。Tasca 等 ${ }^{[5]}$ 制备了 $\mathrm{CuFe}_{2} \mathrm{O}_{4}$ 催化剂, 对丙烷进行催化燃烧处理, 特定的反尖晶石结构使其催化活性大大提高, 其完 全转化温度为 $520^{\circ} \mathrm{C}$ 。Zhou 等 ${ }^{[6]}$ 将 $\mathrm{Co}-\mathrm{Mn}$ 尖晶石结 构负载到活性炭上, $\mathrm{Co}$ 的加入减弱了 $\mathrm{Mn}-\mathrm{O}$ 的化学 键能, 增强了其催化燃烧活性, $235^{\circ} \mathrm{C}$ 时甲苯的转化 率可达 $93.5 \%$ 。 Tang 等 ${ }^{[7]}$ 发现在 Pd/SBA-15 催化剂 中掺杂 $\mathrm{Ni}$, 可将苯的完全转化温度降低 $100^{\circ} \mathrm{C}, \mathrm{NiO}$ 物种可以均匀分散于 SBA-15 表面, PdO-NiO 的协同 作用使催化剂的催化活性明显增强。Zhang 等 ${ }^{[8]}$ 制备 了 $\mathrm{NiFe}_{2} \mathrm{O}_{4}$ 催化剂, 对甲烷的完全转化温度在 $400^{\circ} \mathrm{C}$ 左右，且催化剂使用寿命长。Zawadzki 等 ${ }^{[9]}$ 制备的 $\mathrm{ZnFe}_{2} \mathrm{O}_{4}$ 催化剂在 $406^{\circ} \mathrm{C}$ 时对环已烷的转化率可达 $95 \%$, 转化温度明显低于 $\mathrm{CoAl}_{2} \mathrm{O}_{4}$ 催化剂。 $\mathrm{ZnFe}_{2} \mathrm{O}_{4}$ 催化剂中 $\mathrm{Fe}$ 的 $\mathrm{H}_{2}$ 还原峰明显高于其他催化剂, 结 合有机物在金属氧化物上的氧化还原机理, $\mathrm{Zn}$ 在 $\mathrm{A}$ 位上的取代，增加了其催化活性。除了过渡金属外， 碱金属 $\mathrm{Mg}$ 也可作为 $\mathrm{AFe}_{2} \mathrm{O}_{4}$ 催化剂的 $\mathrm{A}$ 位元素, Shakir 等 ${ }^{[10]}$ 将 $\mathrm{MgFe}_{2} \mathrm{O}_{4}$ 纳米尖晶石结构负载到石墨 烯上, $\mathrm{MgFe}_{2} \mathrm{O}_{4}$ 催化剂表现出较好的催化活性。Jia 等 ${ }^{[11]}$ 制备了 $\mathrm{Au} / \mathrm{MgFe}_{2} \mathrm{O}_{4}$ 催化剂, 应用于 $\mathrm{CO}$ 的催化 氧化, 发现加入 $\mathrm{MgFe}_{2} \mathrm{O}_{4}$ 可以提高 $\mathrm{Au}$ 的催化活性。 虽然 $\mathrm{AFe}_{2} \mathrm{O}_{4}$ 表现出较好的催化活性, 但以往的研
究只针对一种 A 位元素进行分析, 并没有对多种 A 位元素在 VOCs 催化燃烧中进行系统的比较分析。

鉴于此，本工作以甲苯为 VOCs 模拟气，对 $\mathrm{AFe}_{2} \mathrm{O}_{4}(\mathrm{~A}=\mathrm{Cu}, \mathrm{Co}, \mathrm{Ni}, \mathrm{Mg}, \mathrm{Zn})$ 催化剂的催化燃烧活 性进行研究, 并通过 XRD、 $\mathrm{N}_{2}$ 吸附-脱附、SEM、 TEM、 $\mathrm{H}_{2}$-TPR、XPS 等技术分析催化剂的晶体结构、 孔结构、微观结构、氧化还原能力、表面元素组成 等, 系统地探究 $\mathrm{A}$ 位元素对 $\mathrm{AFe}_{2} \mathrm{O}_{4}$ 催化燃烧 VOCs 活性的影响。

\section{1 实验方法}

\section{1 实验试剂}

实验试剂主要有: 三水合硝酸铜 $\left(\mathrm{Cu}\left(\mathrm{NO}_{3}\right)_{2}\right.$. $\left.3 \mathrm{H}_{2} \mathrm{O}\right)$ 、六水合硝酸钴 $\left(\mathrm{Co}\left(\mathrm{NO}_{3}\right)_{2} \cdot 6 \mathrm{H}_{2} \mathrm{O}\right)$ 、六水合硝 酸镁 $\left(\mathrm{Mg}\left(\mathrm{NO}_{3}\right)_{2} \cdot 6 \mathrm{H}_{2} \mathrm{O}\right)$ 、六水合硝酸锌 $\left(\mathrm{Zn}\left(\mathrm{NO}_{3}\right)_{2}\right.$. $\left.6 \mathrm{H}_{2} \mathrm{O}\right)$ 、六水合硝酸镍 $\left(\mathrm{Ni}\left(\mathrm{NO}_{3}\right)_{2} \cdot 6 \mathrm{H}_{2} \mathrm{O}\right)$ 、九水合硝 酸铁 $\left(\mathrm{Fe}\left(\mathrm{NO}_{3}\right)_{3} \cdot 9 \mathrm{H}_{2} \mathrm{O}\right)$ 、一水合柠檬酸 $\left(\mathrm{C}_{6} \mathrm{H}_{8} \mathrm{O}_{7} \cdot \mathrm{H}_{2} \mathrm{O}\right)$ 、 氨水, 以上实验试剂均为分析纯 (Analytical grade, $\mathrm{AR})$, 由国药集团化学试剂有限公司生产。

\section{$1.2 \mathrm{AFe}_{2} \mathrm{O}_{4}$ 催化剂的制备}

通过溶胶一凝胶-自蔓延燃烧法, 以柠檬酸作络 合剂, 制备系列 $\mathrm{AFe}_{2} \mathrm{O}_{4}$ 催化剂。按照摩尔比 $n_{(\mathrm{A})}: n_{(\mathrm{Fe})}=$ $1: 2$ 分别称取 $\mathrm{Cu}\left(\mathrm{NO}_{3}\right)_{2} \cdot 3 \mathrm{H}_{2} \mathrm{O} 、 \mathrm{Co}\left(\mathrm{NO}_{3}\right)_{2} \cdot 6 \mathrm{H}_{2} \mathrm{O}$ 、 $\mathrm{Mg}\left(\mathrm{NO}_{3}\right)_{2} \cdot 6 \mathrm{H}_{2} \mathrm{O} 、 \mathrm{Zn}\left(\mathrm{NO}_{3}\right)_{2} \cdot 6 \mathrm{H}_{2} \mathrm{O} 、 \mathrm{Ni}\left(\mathrm{NO}_{3}\right)_{2} \cdot 6 \mathrm{H}_{2} \mathrm{O}$ 及 $\mathrm{Fe}\left(\mathrm{NO}_{3}\right)_{3} \cdot 9 \mathrm{H}_{2} \mathrm{O}$ 溶于去离子水, 形成金属硝酸盐 前驱溶液 $\mathrm{A}$ 。按照 $n_{\text {(金属离子) }}: n_{\text {(标栖酸) }}=1: 1$, 将一水合 柠檬酸溶于去离子水, 形成柠檬酸前驱溶液 $\mathrm{B}$ 。将 溶液 $\mathrm{B}$ 缓慢加入溶液 $\mathrm{A}$ 中, 用氨水调 $\mathrm{pH}$ 至 $7 \pm 0.5$, 并在 $(70 \pm 5)^{\circ} \mathrm{C}$ 下持续摚拌至溶液呈溶胶状态。然后 溶胶老化 $2 \mathrm{~h}$, 使其形成凝胶, 凝胶在 $130^{\circ} \mathrm{C}$ 下鼓风 干燥 $12 \mathrm{~h}$, 形成干凝胶。将干凝胶研成粉末，置于瓷 元血中, 放入 $300^{\circ} \mathrm{C}$ 马弗炉中加热。干凝胶在高温下 点燃，并自干凝胶表面向内部自蔓延燃烧，燃烧形 成蓬松的树状粉末。将燃烧产物冷却、研磨, 在 $500^{\circ} \mathrm{C}$ 马弗炉中焙烧 $2 \mathrm{~h}$, 制备成粉末状催化剂。将粉末状 催化剂压片和过䇻，选取 $250 \sim 420 \mu \mathrm{m}$ 的颗粒状催 化剂, 密封保存。

\section{$1.3 \mathrm{AFe}_{2} \mathrm{O}_{4}$ 催化燃烧活性测定}

在催化燃烧固定床反应器上测定催化剂的催化 燃烧活性，反应装置如图 1 所示。本实验量取 $5 \mathrm{~mL}(250 \sim 420 \mu \mathrm{m}, 7 \sim 8 \mathrm{~g})$ 催化剂, 并用 $10 \mathrm{~mL}$ 石英 
砂稀释, 将混合物置于催化燃烧反应器床层上。反 应器升温速率 $10^{\circ} \mathrm{C} / \mathrm{min}$, 空气流速 $(500 \pm 5) \mathrm{mL} / \mathrm{min}$, 进气甲苯浓度为 $(300 \pm 50) \mathrm{mg} / \mathrm{m}^{3}$, 反应装置内部压 力 $0.8 \mathrm{MPa}$, 空速为 $6000 / \mathrm{h}$ 。通过 SP-3420 型气相色 谱仪(北京北分瑞利公司)检测进、出口甲苯浓度, 采 用 FID 检测器, 检测器温度 $200^{\circ} \mathrm{C}$, 进样温度 $150^{\circ} \mathrm{C}$, 柱温 $120^{\circ} \mathrm{C}$ 。

\section{$1.4 \mathrm{AFe}_{2} \mathrm{O}_{4}$ 催化剂的表征}

采用 $x^{\prime}$ PertPro MPD 型 $\mathrm{X}$ 射线粉末衍射仪 (PANalytical B V, Holland)对样品进行 X 射线衍射分 析(X-ray diffraction patterns, XRD), 以 $\mathrm{Ni}$ 金属为滤 波片、 $\mathrm{Cu}-\mathrm{K} \alpha$ 为光源, 管电压: $40 \mathrm{kV}$, 管电流: $50 \mathrm{~mA}$, 扫描范围: $2 \theta=5^{\circ} \sim 75^{\circ}$, 扫描速度: $4^{\circ} / \mathrm{min}$ 。

采用 ASAP2020M 型比表面积及微孔物理吸附 分析仪(Micrometrics Instrument Crop, USA)对样品 进行比表面积(Brunauer-Emmett-Teller, BET)及孔结 构分析, 催化剂样品先在 $300^{\circ} \mathrm{C}$ 下抽真空处理 $5 \mathrm{~h}$, 然后在液氮温度 $\left(-196^{\circ} \mathrm{C}\right)$ 下进行 $\mathrm{N}_{2}$ 吸附一脱附实验。

采用 MERLIN Compact 型超高分辨率场发射扫 描电镜(Carl Zeiss Jena, Germany)对样品进行观察, 镜筒内光轴采用 InLens 二次电子探头, EHT: $15 \mathrm{kV}$ 。

采用 JEM-2010 型高分辨率通用型透射电子显 微镜(Japan Electron Optics Laboratory, Japan)对样品 进行观察, 采用 $\mathrm{LaB}_{6}$ 灯丝, 加速电压: $200 \mathrm{kV}$ 。

采用 TP5079 型多用化学吸附仪(Tianjin Xianquan, China)对样品进行氢气程序升温还原分析 $\left(\mathrm{H}_{2}-\mathrm{TPR}\right)$, 称取 $100 \mathrm{mg}$ 催化剂, 首先在 $\mathrm{He}$ 气氛中 $300^{\circ} \mathrm{C}$ 吹扫 $0.5 \mathrm{~h}$, 去除催化剂表面的杂质; 当温度 降至 $80^{\circ} \mathrm{C}$ 时, 开始通入 $\mathrm{H}_{2}$ 进行还原分析。还原温度: 室温 $800^{\circ} \mathrm{C}$, 升温速率: $10^{\circ} \mathrm{C} / \mathrm{min}$ 。

采用 Escalab 250Xi 型 $\mathrm{X}$ 射线光电子能谱仪 (Thermo Fisher Scientific, USA) 对样品进行 XPS 分 析, 采用单色化的 $\mathrm{Al}-\mathrm{K} \alpha$ 为 $\mathrm{X}$ 射线光源, CAE 扫描 模式, 采用 Large Area XL 透镜模式, 束斑大小: $500 \mu \mathrm{m}$, 以表面污染 $\mathrm{C} 1 \mathrm{~s}(284.8 \mathrm{eV})$ 为标准进行能谱 校正。

\section{2 结果与讨论}

\section{$2.1 \mathrm{AFe}_{2} \mathrm{O}_{4}$ 催化燃烧活性研究}

图 2 给出了 $\mathrm{AFe}_{2} \mathrm{O}_{4}(\mathrm{~A}=\mathrm{Cu}, \mathrm{Co}, \mathrm{Ni}, \mathrm{Mg}, \mathrm{Zn})$ 催化 剂在 $100 \sim 400^{\circ} \mathrm{C}$ 对甲苯的催化燃烧活性曲线。由图 2 分析催化剂的起燃温度 $\left(T_{50}\right)$, 发现其对甲苯催化燃 烧活性依次为: $\mathrm{CuFe}_{2} \mathrm{O}_{4}\left(188^{\circ} \mathrm{C}\right)>\mathrm{NiFe}_{2} \mathrm{O}_{4}\left(200^{\circ} \mathrm{C}\right)>$ $\mathrm{CoFe}_{2} \mathrm{O}_{4}\left(223^{\circ} \mathrm{C}\right)>\mathrm{ZnFe}_{2} \mathrm{O}_{4}\left(260^{\circ} \mathrm{C}\right)>\mathrm{MgFe}_{2} \mathrm{O}_{4}\left(275^{\circ} \mathrm{C}\right)$; 分析甲苯完全转化温度 $\left(T_{90}\right)$, 发现催化剂对甲苯催 化燃烧活性依次为: $\mathrm{CuFe}_{2} \mathrm{O}_{4}\left(239^{\circ} \mathrm{C}\right)>\mathrm{CoFe}_{2} \mathrm{O}_{4}\left(257^{\circ} \mathrm{C}\right)>$ $\mathrm{ZnFe}_{2} \mathrm{O}_{4}\left(314^{\circ} \mathrm{C}\right)>\mathrm{MgFe}_{2} \mathrm{O}_{4}\left(350^{\circ} \mathrm{C}\right)>\mathrm{NiFe}_{2} \mathrm{O}_{4}\left(352^{\circ} \mathrm{C}\right)$ 。 所制备的催化剂表现出较高的催化燃烧活性, 且不 同 $\mathrm{A}$ 位元素对 $\mathrm{AFe}_{2} \mathrm{O}_{4}$ 催化燃烧活性影响较大, 其中 $\mathrm{CuFe}_{2} \mathrm{O}_{4}$ 的催化燃烧活性最好, $T_{50}$ 和 $T_{90}$ 远低于其他 催化剂。 $\mathrm{NiFe}_{2} \mathrm{O}_{4}$ 催化剂的 $T_{50}$ 仅高于 $\mathrm{CuFe}_{2} \mathrm{O}_{4}$ 催化 剂 $12^{\circ} \mathrm{C}$, 且明显低于其他催化剂, 但其 $T_{90}$ 则高于 其他催化剂。可见 $\mathrm{NiFe}_{2} \mathrm{O}_{4}$ 催化剂在低温段的催化

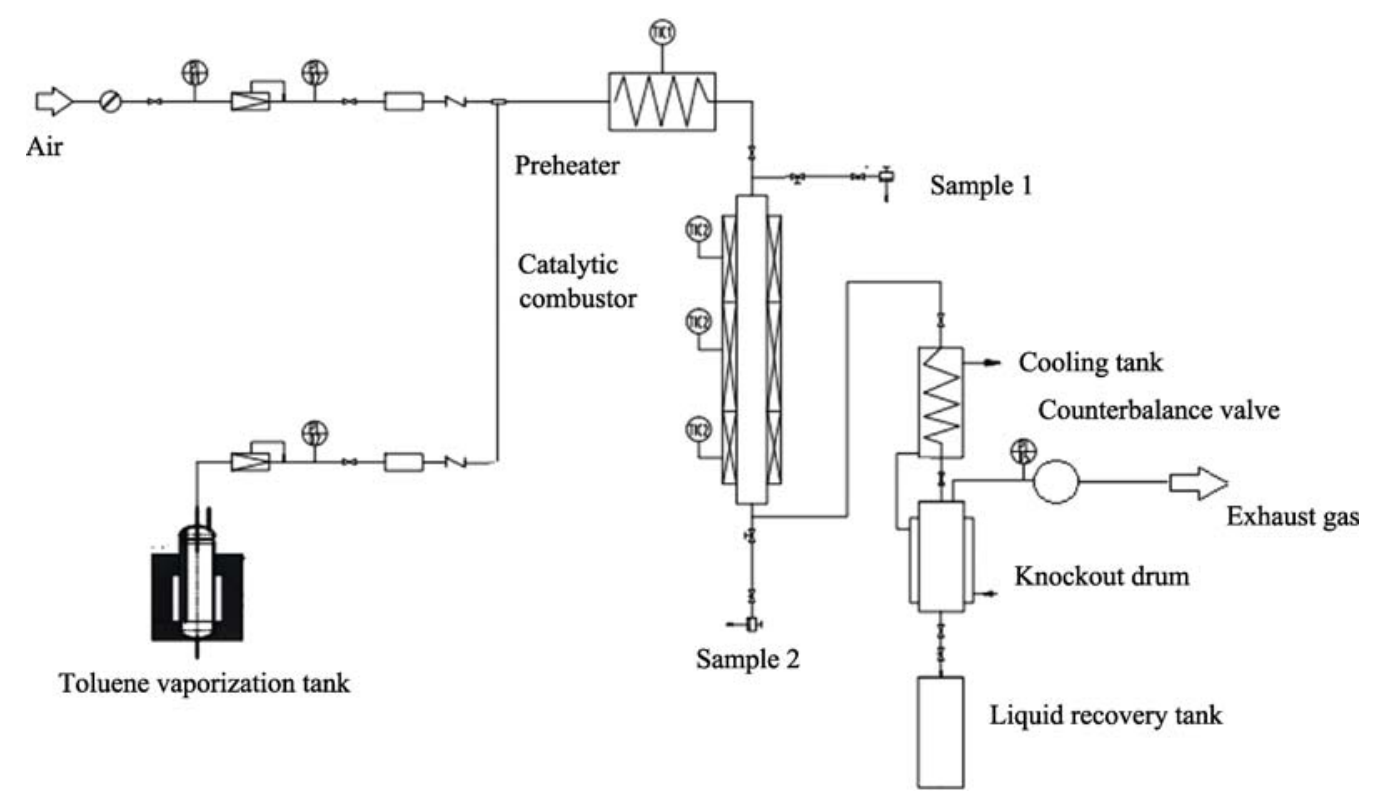

图 1 催化燃烧固定床反应装置流程图

Fig. 1 Flow chart of fixed bed catalytic combustion 


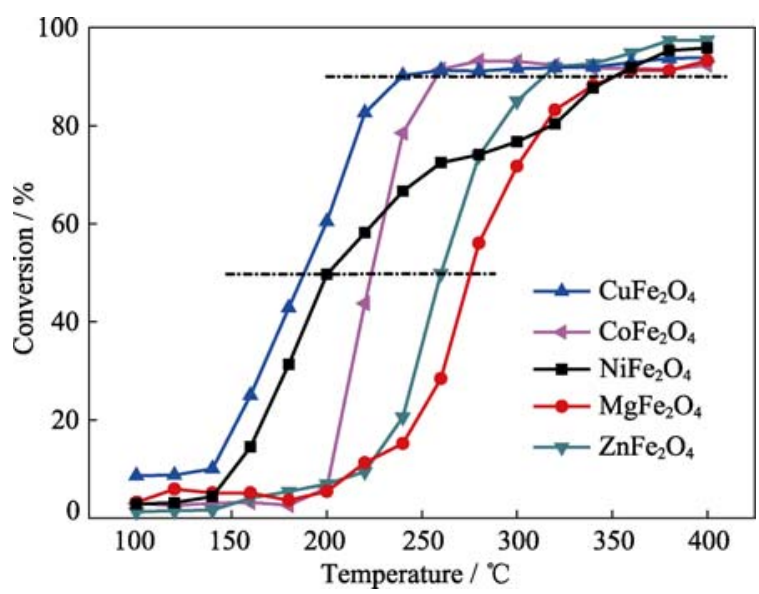

图 $2 \mathrm{AFe}_{2} \mathrm{O}_{4}$ 催化燃烧甲苯曲线

Fig. 2 Catalytic combustion curves of toluene over $\mathrm{AFe}_{2} \mathrm{O}_{4}$

燃烧活性较高, 但在高温段的催化活性较差。 Meshkani 等 ${ }^{[12]}$ 认为元素的电负性越大, 晶格氧的流 动量越大, 催化剂的催化活性越高。 $\mathrm{Liu}$ 等 ${ }^{[13]}$ 认为电 负性通过影响催化剂的离子半径和氧化还原电位, 进而影响催化剂的催化活性, 并发现电负性越大, 晶格氧含量越多, 催化剂的催化活性越好。本实验中 $A$ 位元素的电负性依次为: $\mathrm{Cu}(1.9)>\mathrm{Ni}(1.8)=\mathrm{Co}(1.8)>$ $\mathrm{Zn}(1.6)>\mathrm{Mg}(1.2)$, 与催化剂在 $T_{50}$ 时的催化活性一 致, 说明 $\mathrm{AFe}_{2} \mathrm{O}_{4}$ 催化剂中 $\mathrm{A}$ 位元素的电负性对其催 化活性有一定影响。

\section{$2.2 \mathrm{AFe}_{2} \mathrm{O}_{4}$ 催化剂 $\mathrm{XRD}$ 分析}

图 3 为 $\mathrm{AFe}_{2} \mathrm{O}_{4}$ 的 XRD 图谱, 由图中可以看出, 催化剂均具有明显的尖晶石晶型。在 $\mathrm{CoFe}_{2} \mathrm{O}_{4}$ (JCPDS 22-1086)、 $\mathrm{ZnFe}_{2} \mathrm{O}_{4}$ (JCPDS 82-1042)、 $\mathrm{NiFe}_{2} \mathrm{O}_{4}$ (JCPDS 74-2081)、 $\mathrm{MgFe}_{2} \mathrm{O}_{4}$ (JCPDS 88-1938)晶体中, $\mathrm{Co}^{2+} 、 \mathrm{Ni}^{2+} 、 \mathrm{Mg}^{2+} 、 \mathrm{Zn}^{2+}$ 存在于尖晶石 $\mathrm{A}$ 位正四面体 中, $\mathrm{Fe}^{3+}$ 存在于 $\mathrm{B}$ 位的正八面体中, 为典型正尖晶石 的 Fd-3m 晶群 ${ }^{[14]}$ 。在各催化剂中, 特别是 $\mathrm{NiFe}_{2} \mathrm{O}_{4}$

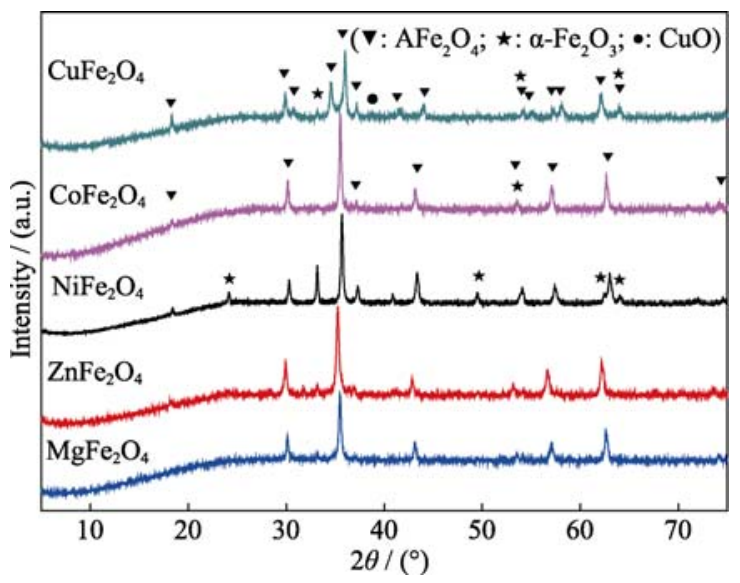

图 $3 \mathrm{AFe}_{2} \mathrm{O}_{4}$ 的 XRD 图谱

Fig. 3 XRD patterns of $\mathrm{AFe}_{2} \mathrm{O}_{4}$
中出现了 $\alpha-\mathrm{Fe}_{2} \mathrm{O}_{3}$ (JCPDS 84-0306)斜方六面体晶系 R-3c 型晶群的(012)、(104)、(024)晶面衍射峰。Said 等 ${ }^{[15]}$ 实验证明, $\alpha-\mathrm{Fe}_{2} \mathrm{O}_{3}$ 结构本身具有一定的催化活 性, $\alpha-\mathrm{Fe}_{2} \mathrm{O}_{3}$ 与 $\mathrm{AFe}_{2} \mathrm{O}_{4}$ 尖晶石物种的协同作用可以 增加催化剂的表面活性位。另外, $\alpha-\mathrm{Fe}_{2} \mathrm{O}_{3}$ 与 $\mathrm{AFe}_{2} \mathrm{O}_{4}$ 尖晶石物种中 $\mathrm{Fe}^{3+}-\mathrm{Fe}^{2+}$ 离子之间的电子转移, 使催 化剂一直处于亚稳定状态, 产生较多晶格缺陷, 这 是 $\mathrm{AFe}_{2} \mathrm{O}_{4}$ 催化剂具有较高催化燃烧活性的重要原 因, 也是 $\mathrm{NiFe}_{2} \mathrm{O}_{4}$ 在低温段催化燃烧活性高的重要 原因。在 $\mathrm{CuFe}_{2} \mathrm{O}_{4}$ (JCPDS 72-1174) 晶体中, 由于晶 体的 Jahn-Teller 效应, A 位上的 $\mathrm{Cu}^{2+}$ 发生正四面体畸 变, 转向 $\mathrm{B}$ 位正八面体中, 两个 $\mathrm{Fe}$ 离子则分别存在 于正四面体和正八面体中，形成反式尖晶石结构的 I4 1 /amd 晶群, 这符合晶体场稳定能相关理论, 与 Tasca 等 $^{[5]}$ 研究结果一致。 $\mathrm{Fe}$ 离子在正四面体和正 八面体中出现, 更有利于 $\mathrm{Fe}^{3+}-\mathrm{Fe}^{2+}$ 离子之间的载流 电子交换, 提高了催化剂的晶格畸变能, 从而提高 了其催化燃烧活性。另外, 与其他正尖晶石催化剂 相比, 反尖晶石的存在, 使 $\mathrm{CuFe}_{2} \mathrm{O}_{4}$ 的晶格体积仅 为 $0.294 \mathrm{~nm}^{3}$, 远低于其他催化剂, 从而提高了 $\mathrm{CuFe}_{2} \mathrm{O}_{4}$ 催化剂的催化燃烧活性。在 $\mathrm{CuFe}_{2} \mathrm{O}_{4}$ 的 XRD 衍射峰中, 还出现了 $\mathrm{CuO}(J C P D S$ 89-5899)单 斜晶系 Cc 型晶群(111) 晶面的特征峰。Cheng 等 ${ }^{[16]}$ 研究发现, 表面分散的 $\mathrm{CuO}$ 对催化剂的催化活性有 一定提高, 且 $\alpha-\mathrm{Fe}_{2} \mathrm{O}_{3}$ 与 $\mathrm{CuO}$ 物种的协同作用可以 使 $\mathrm{CuO}$ 进一步处于分散状态, 从而提高 $\mathrm{CuFe}_{2} \mathrm{O}_{4}$ 催 化剂的催化燃烧活性。

通过 Scherre 公式计算 $\mathrm{CoFe}_{2} \mathrm{O}_{4} 、 \mathrm{ZnFe}_{2} \mathrm{O}_{4}$ 、 $\mathrm{NiFe}_{2} \mathrm{O}_{4} 、 \mathrm{MgFe}_{2} \mathrm{O}_{4}$ 在(311) 晶面处以及 $\mathrm{CuFe}_{2} \mathrm{O}_{4}$ 在 (211)晶面处的晶格尺寸, 结果列于表 1 。从表 1 中 可以看出, $\mathrm{CuFe}_{2} \mathrm{O}_{4} 、 \mathrm{CoFe}_{2} \mathrm{O}_{4} 、 \mathrm{ZnFe}_{2} \mathrm{O}_{4} 、 \mathrm{NiFe}_{2} \mathrm{O}_{4}$ 、 $\mathrm{MgFe}_{2} \mathrm{O}_{4}$ 的晶格尺寸差距不大，该计算结果与 Rezlescu 等 ${ }^{[17]}$ 通过溶胶一凝胶-自蔓延燃烧法制备的 铁酸盐尖晶石材料的晶格尺寸相近。结合图 2 分析, 发现晶格尺寸与催化剂的催化燃烧活性没有明显的 相关性，可见颗粒状催化剂的晶格尺寸对其非均相 催化过程的影响较小。

\section{$2.3 \mathrm{AFe}_{2} \mathrm{O}_{4}$ 催化剂孔结构分析}

图 4 为 $\mathrm{AFe}_{2} \mathrm{O}_{4}$ 的孔分布曲线, 表 1 为 $\mathrm{AFe}_{2} \mathrm{O}_{4}$ 的孔结构参数, 图 5 为 $\mathrm{CuFe}_{2} \mathrm{O}_{4}$ 的 SEM 和 TEM 照 片。由图 4 及表 1 可以看出, 催化剂平均孔径为 19.8 23.0 nm, 以 $2.5 \mathrm{~nm}$ 左右的介孔结构为主, 并 有微孔和大孔结构存在, 呈以介孔为主的多级孔结 构。Koekkoek 等 ${ }^{[18]}$ 研究发现多级孔结构可以增加非 均相催化的传质速率，并且，多级孔结构为不同 VOCs 气体在催化剂表面的传递创造了条件。从图 5 
表 $1 \mathrm{AFe}_{2} \mathrm{O}_{4}$ 的晶体结构及孔结构参数

Table 1 Crystalline and porous structure parameters of $\mathrm{AFe}_{2} \mathrm{O}_{4}$

\begin{tabular}{ccccccc}
\hline Sample & $S_{\mathrm{BET}} /\left(\mathrm{m}^{2} \cdot \mathrm{g}^{-1}\right)$ & Pore size $/ \mathrm{nm}$ & Pore volume $/\left(\mathrm{cm}^{3} \cdot \mathrm{g}^{-1}\right)$ & Space group & Crystallite size $/ \mathrm{nm}$ & Cell volume $/ \mathrm{nm}^{3}$ \\
\hline $\mathrm{MgFe}_{2} \mathrm{O}_{4}$ & 21.5 & 19.8 & 0.11 & $\mathrm{Fd}-3 \mathrm{~m}$ & 41.6 & 0.59057 \\
$\mathrm{ZnFe}_{2} \mathrm{O}_{4}$ & 19.4 & 22.4 & 0.12 & $\mathrm{Fd}-3 \mathrm{~m}$ & 31.5 & 0.60265 \\
$\mathrm{CuFe}_{2} \mathrm{O}_{4}$ & 10.5 & 23.0 & 0.06 & $\mathrm{I} 4{ }_{1} / \mathrm{amd}$ & 44.5 & 0.29402 \\
$\mathrm{CoFe}_{2} \mathrm{O}_{4}$ & 15.1 & 19.7 & 0.07 & Fd-3m & 46.3 & 0.59099 \\
$\mathrm{NiFe}_{2} \mathrm{O}_{4}$ & 5.4 & 21.9 & 0.03 & Fd-3m & 44.0 & 0.57966 \\
\hline
\end{tabular}

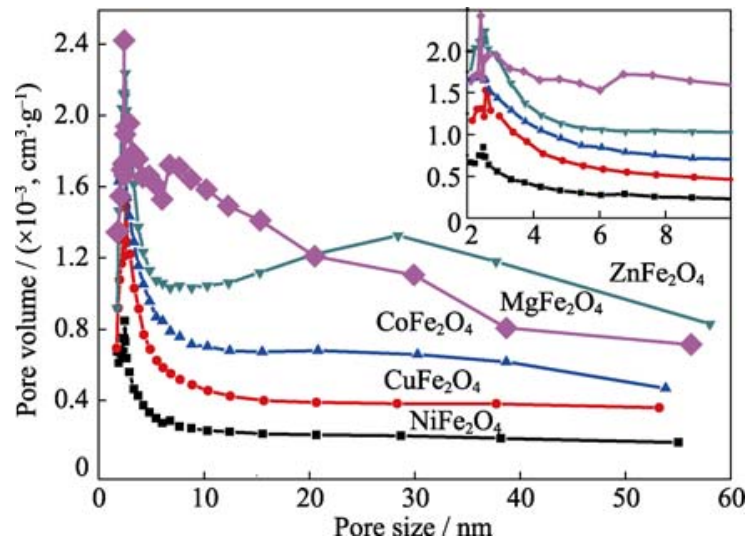

图 $4 \mathrm{AFe}_{2} \mathrm{O}_{4}$ 的孔分布曲线

Fig. 4 Pore size distribution curves of $\mathrm{AFe}_{2} \mathrm{O}_{4}$

可以看出, 催化剂呈明显片状结构, 这与 Surblé 等 ${ }^{[19]}$ 的研究结果一致。片状结构的存在, 有利于催化剂 表面活性位的分散, 从而提高催化剂与 VOCs 气体 的结合, 提高催化燃烧活性。由于各催化剂孔径的 差异较小, 且与催化剂的活性没有明显相关性, 可 见孔径对催化剂的催化燃烧活性影响较小。

通过孔结构参数计算, 各催化剂的比表面积如 表 1 所示。 $\mathrm{CuFe}_{2} \mathrm{O}_{4} 、 \mathrm{CoFe}_{2} \mathrm{O}_{4} 、 \mathrm{ZnFe}_{2} \mathrm{O}_{4} 、 \mathrm{MgFe}_{2} \mathrm{O}_{4}$ 比表面积与催化活性相关性较小, 说明当比表面积 相近时, 比表面积不是颗粒状催化剂催化活性的主
要影响因素; 但比表面积差距较大时, 它对催化剂 催化活性的影响较大。 $\mathrm{NiFe}_{2} \mathrm{O}_{4}$ 的比表面积仅为 $5.4 \mathrm{~m}^{2} / \mathrm{g}$, 远低于其他催化剂, 这严重限制了甲苯在 $\mathrm{NiFe}_{2} \mathrm{O}_{4}$ 表面的结合, 甲苯气体不能与催化剂表面 活性位点充分接触, 故在高温段表现出较差的催化 活性。

\section{$2.4 \mathrm{CuFe}_{2} \mathrm{O}_{4}$ 催化剂 XPS 分析}

图 6 为 $\mathrm{CuFe}_{2} \mathrm{O}_{4}$ 的 XPS 谱图, 由图可见, 在催 化剂表面分别出现了 $\mathrm{O} 1 \mathrm{~s}(545 \sim 525 \mathrm{eV}) 、 \mathrm{Fe} 2 \mathrm{p}$ (740 $700 \mathrm{eV}) 、 \mathrm{Cu} 2 \mathrm{p}(953 \sim 933 \mathrm{eV})$ 的光电子能谱峰, 这与 Yadav 等 ${ }^{[20]}$ 制备的尖晶石材料的 XPS 谱图一致。对 $\mathrm{CuFe}_{2} \mathrm{O}_{4}$ 的 XPS 谱图进行半定量分峰, 发现 $n(\mathrm{Cu})$ : $n(\mathrm{Fe})$ 为 0.29:0.71, 小于 $\mathrm{CuFe}_{2} \mathrm{O}_{4}$ 分子式中的比例。 结合 XRD 结果分析, 这可能是由于在焙烧过程中, 较多 $\alpha-\mathrm{Fe}_{2} \mathrm{O}_{3}$ 晶体存在于催化剂表面，使催化剂表 面 $\mathrm{Fe}$ 的比例增加。

催化剂中 $\mathrm{O}$ 的种类和含量会影响催化剂的催化 活性，通过 O1s 的 XPS 分析(图 6b), 发现在催化剂 表面有三种氧物种: (1) $529.5 \mathrm{eV}$ 对应晶格氧 $\mathrm{O}_{\mathrm{latt}}$ $\left(\mathrm{CuFe}_{2} \mathrm{O}_{4}, \alpha-\mathrm{Fe}_{2} \mathrm{O}_{3} \text { 晶格上的 } \mathrm{O}\right)^{[21]}$; (2) $530.8 \mathrm{eV}$ 对应 表面亲电子氧和氧空位 $\mathrm{O}_{\mathrm{ele}}\left(\mathrm{OH}^{-}, \mathrm{C}-\mathrm{O}, \mathrm{O}-\mathrm{C}=\mathrm{O}\right)$; (3) $532.2 \mathrm{eV}$ 对应吸附氧 $\mathrm{O}_{\mathrm{ads}}\left(\mathrm{H}_{2} \mathrm{O} \text { 等 }\right)^{[22]}$ 。其中 $\mathrm{O}_{\text {ele }}$ 以氧空位为主, 氧空位可以促进非均相反应中氧的
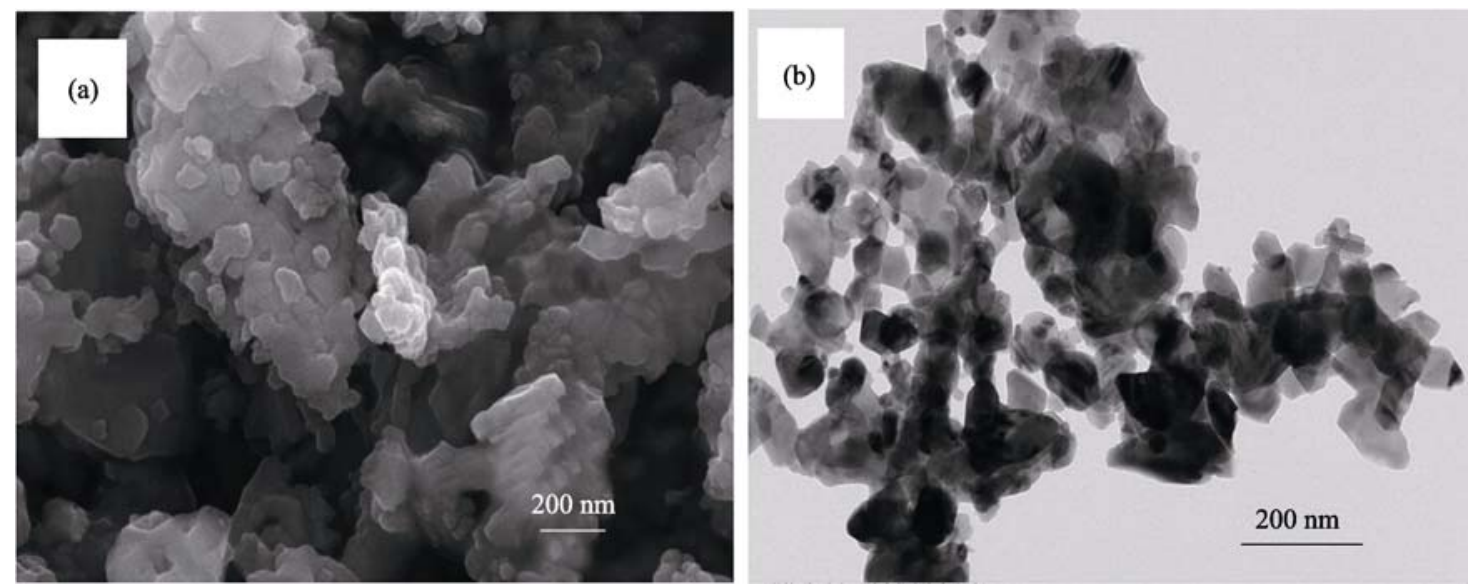

图 $5 \mathrm{CuFe}_{2} \mathrm{O}_{4}$ 的 $\operatorname{SEM}(\mathrm{a})$ 和 TEM(b)照片

Fig. 5 SEM (a) and TEM (b) images of $\mathrm{CuFe}_{2} \mathrm{O}_{4}$ 

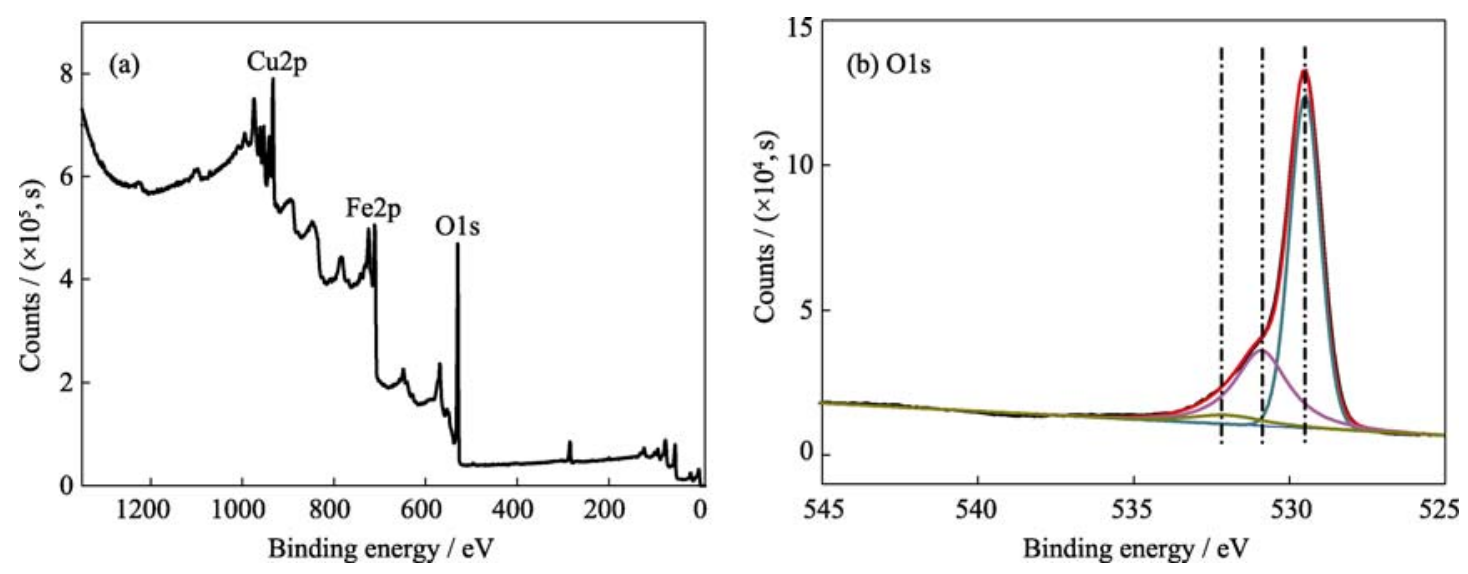

图 $6 \mathrm{CuFe}_{2} \mathrm{O}_{4}$ 的 XPS 全谱图(a)和 $\mathrm{O} 1 \mathrm{~s}$ 的 XPS 谱图(b)

Fig. 6 Full XPS spectrum (a) and O1s spectrum (b) of $\mathrm{CuFe}_{2} \mathrm{O}_{4}$

迁移能力, 是影响催化剂的催化燃烧活性的重要因 素之一 ${ }^{[23]}$ 。本实验制备的催化剂 $\mathrm{O}_{\mathrm{ele}}$ 占所有氧物种 的 36\%, 与 $\mathrm{WANG}$ 等 ${ }^{[24]}$ 制备的催化剂中 $\mathrm{O}_{\text {ele }}$ 的比例 一致, 说明催化剂中氧空位含量适中。

\section{$2.5 \mathrm{AFe}_{2} \mathrm{O}_{4}$ 催化剂 $\mathrm{H}_{2}-\mathrm{TPR}$ 分析}

图 7 为 $\mathrm{AFe}_{2} \mathrm{O}_{4}$ 的 $\mathrm{H}_{2}$-TPR 图谱, 由图 7 可知, $\mathrm{AFe}_{2} \mathrm{O}_{4}$ 催化剂在 $455^{\circ} \mathrm{C}\left(\alpha\right.$ 峰)和 $633^{\circ} \mathrm{C}$ ( $\beta$ 峰) 分别出现 了 $\mathrm{Fe}^{3+} \rightarrow \mathrm{Fe}^{3+}+\mathrm{Fe}^{2+}\left(\mathrm{Fe}_{2} \mathrm{O}_{3} \rightarrow \mathrm{AFe}_{2} \mathrm{O}_{4}\right)$ 和 $\mathrm{Fe}^{3+}+\mathrm{Fe}^{2+} \rightarrow$ $\mathrm{Fe}^{2+} \rightarrow \mathrm{Fe}^{0}\left(\mathrm{AFe}_{2} \mathrm{O}_{4} \rightarrow \mathrm{FeO} \rightarrow \mathrm{Fe}\right)$ 还原峰 ${ }^{[12,25]}$, 不同的 $\mathrm{A}$ 位元素对 $\mathrm{Fe}$ 的 $\mathrm{H}_{2}$ 还原峰的影响较大。其中 $\mathrm{CuFe}_{2} \mathrm{O}_{4}$ 、 $\mathrm{CoFe}_{2} \mathrm{O}_{4} 、 \mathrm{ZnFe}_{2} \mathrm{O}_{4} 、 \mathrm{MgFe}_{2} \mathrm{O}_{4}$ 催化剂 $\alpha$ 峰的面积明 显小于 $\beta$ 峰, 说明几种催化剂以 $\mathrm{AFe}_{2} \mathrm{O}_{4}$ 尖晶石结构 为主。而 $\mathrm{NiFe}_{2} \mathrm{O}_{4}$ 中 $\alpha$ 峰的面积大于 $\beta$ 峰的面积, 说 明其中 $\mathrm{Fe}_{2} \mathrm{O}_{3}$ 的含量较多, 这与 XRD 分析结果一致。

$\mathrm{AFe}_{2} \mathrm{O}_{4}$ 催化剂中, $\mathrm{A}$ 位元素的 $\mathrm{H}_{2}$ 还原峰面积和 位置与催化活性关系较大。 $185^{\circ} \mathrm{C}$ 和 $289^{\circ} \mathrm{C}$ 时, 在 $\mathrm{CuFe}_{2} \mathrm{O}_{4}$ 的 $\mathrm{H}_{2}$-TPR 曲线出现了高度分散的 $\mathrm{Cu}^{2+} \rightarrow$ $\mathrm{Cu}^{1+}$ 和结晶中的 $\mathrm{Cu}^{2+} \rightarrow \mathrm{Cu}^{1+}$ 的还原峰 ${ }^{[26]}$ 。 $\mathrm{Cu}$ 的还原 峰温度明显低于其他 $\mathrm{A}$ 位元素, 且 $\mathrm{Cu}$ 的还原峰面

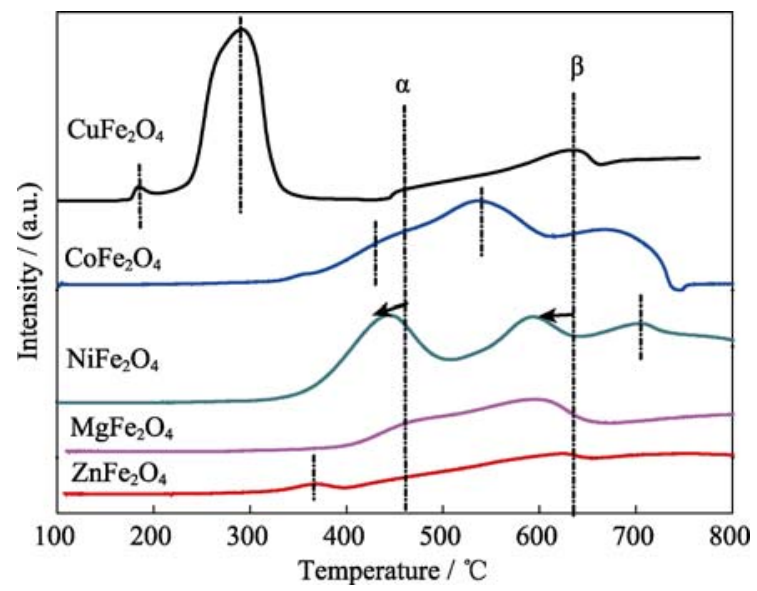

图 $7 \mathrm{AFe}_{2} \mathrm{O}_{4}$ 的 $\mathrm{H}_{2}$-TPR 图谱

Fig. $7 \mathrm{H}_{2}$-TPR spectra of $\mathrm{AFe}_{2} \mathrm{O}_{4}$
积明显大于其他催化剂。结合 XRD 分析, 由于 Jahn-Teller 效应, $\mathrm{CuFe}_{2} \mathrm{O}_{4}$ 形成了反尖晶石晶型。 Meshkani 等 ${ }^{[12]}$ 认为, 反尖晶石结构的出现, 改变了 催化剂中 $\mathrm{Cu}$ 与 $\mathrm{Fe}$ 所占的亚晶格位置, 进而影响催 化剂的 $\mathrm{H}_{2}$ 还原峰面积和还原温度, 这是本研究中 $\mathrm{CuFe}_{2} \mathrm{O}_{4}$ 催化剂的催化活性明显高于其他催化剂的 重要原因。 $430^{\circ} \mathrm{C}$ 和 $540^{\circ} \mathrm{C}$ 时, $\mathrm{CoFe}_{2} \mathrm{O}_{4}$ 的 $\mathrm{H}_{2}$-TPR 曲线 中出现了 $\mathrm{Co}^{3+}+\mathrm{Co}^{2+} \rightarrow \mathrm{Co}^{2+}$ 和 $\mathrm{Co}^{2+} \rightarrow \mathrm{Co}^{0}$ 还原峰 ${ }^{[9,27]}$ 。 但根据 XRD 分析, 几乎不存在 $\mathrm{Co}_{3} \mathrm{O}_{4}$, 可能由于其 处于高度分散状态且含量较少, 故该还原峰的面积 非常小。在 $705^{\circ} \mathrm{C}$ 时, $\mathrm{NiFe}_{2} \mathrm{O}_{4}$ 的 $\mathrm{H}_{2}$-TPR 曲线出现了 较高的还原峰, 对应 $\mathrm{Ni}^{2+} \rightarrow \mathrm{Ni}^{0}$ 的还原峰 ${ }^{[28]}$ 。但该还 原峰的还原温度远高于 $\mathrm{Cu} 、 \mathrm{Zn}$ 和 $\mathrm{Co}$, 这可能是 $\mathrm{NiFe}_{2} \mathrm{O}_{4}$ 在高温段催化活性差的原因之一。在 $\mathrm{ZnFe}_{2} \mathrm{O}_{4}$ 和 $\mathrm{MgFe}_{2} \mathrm{O}_{4}$ 中, 处于正四面体中的 $\mathrm{Mg}^{2+}$ 和 $\mathrm{Zn}^{2+}$ 很难被还原 ${ }^{[29]}$, 因此在 $\mathrm{MgFe}_{2} \mathrm{O}_{4}$ 中没有出现 $\mathrm{Mg}^{2+}$ 的还原峰。在 $\mathrm{ZnFe}_{2} \mathrm{O}_{4}$ 的 $\mathrm{H}_{2}-\mathrm{TPR}$ 曲线中, $370^{\circ} \mathrm{C}$ 时出现了 $\mathrm{Zn}^{2+} \rightarrow \mathrm{Zn}^{0}$ 的还原峰 ${ }^{[30]}$, 但还原峰面积较 小, 这也是 $\mathrm{MgFe}_{2} \mathrm{O}_{4}$ 和 $\mathrm{ZnFe}_{2} \mathrm{O}_{4}$ 的催化燃烧活性差 的重要原因。

\section{3 结论}

采用溶胶-凝胶-自蔓延燃烧法制备了纳米尖 晶石型 $\mathrm{AFe}_{2} \mathrm{O}_{4}(\mathrm{~A}=\mathrm{Cu}, \mathrm{Co}, \mathrm{Ni}, \mathrm{Mg}, \mathrm{Zn})$, 研究 $\mathrm{A}$ 位元 素对 $\mathrm{AFe}_{2} \mathrm{O}_{4}$ 的影响, 主要得到以下结论:

1) $\mathrm{AFe}_{2} \mathrm{O}_{4}$ 催化剂为片状尖晶石晶体, 催化剂的 晶格尺寸对其催化活性影响不大; A 位元素的电负 性对 $\mathrm{AFe}_{2} \mathrm{O}_{4}$ 的催化活性有较大影响, 电负性越高, 催化活性越好。另外, 催化剂以介孔为主的多级孔 结构为 VOCs 的催化燃烧提供了更多的活性位。

2) $\mathrm{CuFe}_{2} \mathrm{O}_{4}$ 中, 由于特定的反 $\mathrm{CuFe}_{2} \mathrm{O}_{4}$ 尖晶石 结构、 $\mathrm{CuO}$ 与 $\alpha-\mathrm{Fe}_{2} \mathrm{O}_{3}$ 三者之间的协同作用, 使催 
化剂表现出更多的表面活性位。 $\mathrm{Cu}$ 的 $\mathrm{H}_{2}$ 还原温度 明显低于其他 $\mathrm{A}$ 位元素, 耗 $\mathrm{H}_{2}$ 量明显高于其他催化 剂, 同时含有大量的氧空位 $\mathrm{O}_{\text {ele }}$ 。

\section{参考文献:}

[1] JUITH VIJAYA J, KENNEDY L JOHN, SEKARAN G, et al. Preparation and VOC gas sensing properties of $\operatorname{Sr}($ II)-added copper aluminate spinel composites. Sensors and Actuators B: Chemical, 2008, 134(2): 604-612.

[2] KARATUM OSMAN, DESHUSSES MARC A. A comparative study of dilute VOCs treatment in a non-thermal plasma reactor. Chemical Engineering Journal, 2016, 294: 308-315.

[3] ZHANG ZHIXIANG, JIANG ZHENG, SHANGGUAN WENFENG. Low-temperature catalysis for VOCs removal in technology and application: a state-of-the-art review. Catalysis Today, 2016, 264: 270-278.

[4] FENG SHAOJIE, YANG WU, WANG ZHONGBING. Synthesis of porous $\mathrm{NiFe}_{2} \mathrm{O}_{4}$ microparticles and its catalytic properties for methane combustion. Materials Science and Engineering: B, 2011, 176(18): 1509-1512.

[5] TASCA JULIA E, QUINCOCES CLAUDIA E, LAVAT ARACELI, et al. Preparation and characterization of $\mathrm{CuFe}_{2} \mathrm{O}_{4}$ bulk catalysts. Ceramics International, 2011, 37(3): 803-812.

[6] ZHOU GUILIN, HE XIAOLING, LIU SHENG, et al. Phenyl VOCs catalytic combustion on supported $\mathrm{CoMn} / \mathrm{AC}$ oxide catalyst. Journal of Industrial and Engineering Chemistry, 2015, 21: 932-941.

[7] TANG WENXIANG, DENG YUZHOU, CHEN YUNFA. Promoting effect of acid treatment on Pd-Ni/SBA-15 catalyst for complete oxidation of gaseous benzene. Catalysis Communications, 2017, 89: 86-90.

[8] ZHANG SHIRAN, SHAN JUNJUN, NIE LONGHUI, et al. In situ studies of surface of $\mathrm{NiFe}_{2} \mathrm{O}_{4}$ catalyst during complete oxidation of methane. Surface Science, 2016, 648: 156-162.

[9] ZAWADZKI MIROSŁAW, OKAL JANINA. Effect of Co and Fe substitution on catalytic VOCs removal on zinc aluminate. Catalysis Today, 2015, 257: 136-143.

[10] SHAKIR IMRAN, SARFRAZ MANSOOR, ALI ZAHID, et al. Magnetically separable and recyclable graphene- $\mathrm{MgFe}_{2} \mathrm{O}_{4}$ nanocomposites for enhanced photocatalytic applications. Journal of Alloys and Compounds, 2016, 660: 450-455.

[11] JIA CHUN-JIANG, LIU YONG, SCHWICKARDI MANFRED, et al. Small gold particles supported on $\mathrm{MgFe}_{2} \mathrm{O}_{4}$ nanocrystals as novel catalyst for $\mathrm{CO}$ oxidation. Applied Catalysis a: General, 2010, 386(1/2): 94-100.

[12] MESHKANI FERESHTEH, REZAEI MEHRAN. Preparation of nanocrystalline metal $(\mathrm{Cr}, \mathrm{Al}, \mathrm{Mn}, \mathrm{Ce}, \mathrm{Ni}, \mathrm{Co}$ and $\mathrm{Cu})$ modified ferrite catalysts for the high temperature water gas shift reaction. Renewable Energy, 2015, 74: 588-598.

[13] LIU YI, WEN CUN, GUO YUN, et al. Modulated CO oxidation activity of M-doped ceria $(\mathrm{M}=\mathrm{Cu}, \mathrm{Ti}, \mathrm{Zr}$, and $\mathrm{Tb})$ : role of the pauling electronegativity of M. Journal of Physical Chemistry C, 2010, 114(21): 9889-9897.

[14] REZLESCU NICOLAE, REZLESCU ELENA, DORIN POPA PAUL, et al. Some nanograined ferrites and perovskites for catalytic combustion of acetone at low temperature. Ceramics International, 2015, 41(3): 4430-4437.

[15] SAID ABD EL-AZIZ A, ABD EL-WAHAB MOHAMED M M, GODA MOHAMED N. Synthesis and characterization of pure and
( $\mathrm{Ce}, \mathrm{Zr}, \mathrm{Ag}$ ) doped mesoporous $\mathrm{CuO}-\mathrm{Fe}_{2} \mathrm{O}_{3}$ as highly efficient and stable nanocatalysts for $\mathrm{CO}$ oxidation at low temperature. Applied Surface Science, 2016, 390: 649-665.

[16] CHENG TAO, FANG ZHIYONG, HU QIXIU, et al. Lowtemperature $\mathrm{CO}$ oxidation over $\mathrm{CuO} / \mathrm{Fe}_{2} \mathrm{O}_{3}$ catalysts. Catalysis Communications, 2007, 8(7): 1167-1171.

[17] REZLESCU N, REZLESCU E, POPA P D, et al. Preparation and characterization of spinel-type $\mathrm{MeFe}_{2} \mathrm{O}_{4}(\mathrm{Me}=\mathrm{Cu}, \mathrm{Cd}, \mathrm{Ni}$ and $\mathrm{Zn})$ for catalyst applications. Materials Chemistry and Physics, 2013, 137(3): 922-927.

[18] KOEKKOEK ARJAN J J, TEMPELMAN CHRISTIAAN H L, DEGIRMENCI VOLKAN, et al. Hierarchical zeolites prepared by organosilane templating: a study of the synthesis mechanism and catalytic activity. Catalysis Today, 2011, 168(1): 96-111.

[19] SURBLÉ SUZY, GOSSET DOMINIQUE, DOLLÉ MICKAËL, et al. Rapidly synthesis of nanocrystalline $\mathrm{MgIn}_{2} \mathrm{O}_{4}$ spinel using combustion and solid state chemistry. Solid State Sciences, 2011, 13(1): 42-48.

[20] YADAV RAGHVENDRA SINGH, HAVLICA JAROMIR, HNATKO MIROSLAV, et al. Magnetic properties of $\mathrm{Co}_{1-x} \mathrm{Zn}_{x} \mathrm{Fe}_{2} \mathrm{O}_{4}$ spinel ferrite nanoparticles synthesized by starch-assisted $\mathrm{Sol}^{-} \mathrm{Gel}$ autocombustion method and its ball milling. Journal of Magnetism and Magnetic Materials, 2015, 378: 190-199.

[21] ZHANG ZHAOLIANG, HAN DONG, WEI SHAOJIE, et al. Determination of active site densities and mechanisms for soot combustion with $\mathrm{O}_{2}$ on Fe-doped $\mathrm{CeO}_{2}$ mixed oxides. Journal of $\mathrm{Ca}$ talysis, 2010, 276(1): 16-23.

[22] BHARGAVA G, GOUZMAN I, CHUN C M, et al. Characterization of the "native" surface thin film on pure polycrystalline iron: a high resolution XPS and TEM study. Applied Surface Science, 2007, 253(9): 4322-4329.

[23] CAO HONGYAN, LI XIAOSHUANG, CHEN YAOQIANG, et al. Effect of loading content of copper oxides on performance of $\mathrm{Mn}-\mathrm{Cu}$ mixed oxide catalysts for catalytic combustion of benzene. Journal of Rare Earths, 2012, 30(9): 871-877.

[24] WANG YU, JIA AI-PIN, LUO MENG-FEI, et al. Highly active spinel type $\mathrm{CoCr}_{2} \mathrm{O}_{4}$ catalysts for dichloromethane oxidation. Applied Catalysis B: Environmental, 2015, 165: 477-486.

[25] ZHU L, ZHONG Z, YANG $\mathrm{H}$, et al. Comparison study of $\mathrm{Cu}-\mathrm{Fe}-\mathrm{Ti}$ and $\mathrm{Co}-\mathrm{Fe}-\mathrm{Ti}$ oxide catalysts for selective catalytic reduction of $\mathrm{NO}$ with $\mathrm{NH}_{3}$ at low temperature. J. Colloid Interface Sci, 2016, 478: 11-21.

[26] DJINOVIĆ PETAR, BATISTA JURKA, PINTAR ALBIN. Calcination temperature and $\mathrm{CuO}$ loading dependence on $\mathrm{CuO}-\mathrm{CeO}_{2}$ catalyst activity for water-gas shift reaction. Applied Catalysis a: General, 2008, 347(1): 23-33.

[27] WANG QING, PENG YUE, FU JIE, et al. Synthesis, characterization, and catalytic evaluation of $\mathrm{Co}_{3} \mathrm{O}_{4} / \gamma-\mathrm{Al}_{2} \mathrm{O}_{3}$ as methane combustion catalysts: significance of $\mathrm{Co}$ species and the redox cycle. Applied Catalysis B: Environmental, 2015, 168-169: 42-50.

[28] HOSSEINI S. A, NIAEI A, SALARI D, et al. Nanocrystalline $\mathrm{AMn}_{2} \mathrm{O}_{4}(\mathrm{~A}=\mathrm{Co}, \mathrm{Ni}, \mathrm{Cu})$ spinels for remediation of volatile organic compounds-synthesis, characterization and catalytic performance. Ceramics International, 2012, 38(2): 1655-1661.

[29] HU JIANAN, ZHAO WENYAN, HU RUISHENG, et al. Catalytic activity of spinel oxides $\mathrm{MgCr}_{2} \mathrm{O}_{4}$ and $\mathrm{CoCr}_{2} \mathrm{O}_{4}$ for methane combustion. Materials Research Bulletin, 2014, 57: 268-273.

[30] DEERATTRAKUL VARISARA, DITTANET PEERAPAN, SAWANGPHRUK MONTREE, et al. $\mathrm{CO}_{2}$ hydrogenation to methanol using $\mathrm{Cu}-\mathrm{Zn}$ catalyst supported on reduced graphene oxide nanosheets. Journal of $\mathrm{CO}_{2}$ Utilization, 2016, 16: 104-113. 